\title{
Lateral Pedicle Graft for management of isolated gingival recession - A case report
}

\author{
Surendra Singh ${ }^{1, *}$, Neetu Rani ${ }^{2}$, Anjani K. Pathak ${ }^{3}$, Nandlal ${ }^{4}$ \\ ${ }^{1}$ Senior Resident, ${ }^{2}$ Reader, ${ }^{3}$ Assistant Professor, ${ }^{4}$ Professor \& HOD, Dept. of Periodontology, Faculty of Dental Sciences, \\ 1,3,4 King George's Medical University, Lucknow, Uttar Pradesh, ${ }^{2}$ Sardar Patel Post Graduate Institute of Dental \& Medical \\ Sciences, Lucknow, Uttar Pradesh, India
}

*Corresponding Author:

Email: surendraagra29@gmail.com

\begin{abstract}
Gingival recession is the apical shift of the marginal gingiva from its normal position on the crown of the tooth to levels on the root surface beyond the cemento-enamel junction.It is a widespread clinical finding in adults with more than 50\% of the population having one or additional sites with gingival recession of $1 \mathrm{~mm}$ or more and its severity increases with age. Root surfaces exposure in oral environment can cause root hypersensitivity, root caries and create esthetic problems. The pedicle graft is simple and less time-consuming surgical procedure with excellent results, without involving second surgical site for donor harvesting. In this case report the Miller's Class-II isolated gingival recession defect was treated with lateral pedicle graft and postoperative clinical outcome showed stable \& significant root coverage.
\end{abstract}

Keywords: Gingival recession, Lateral pedical graft, laterally positioned flap, Root coverage, Mucogingival surgery.

\section{Introduction}

The gingival recession, sometimes called root exposure is defined as "the apical migration of gingival margin beyond the cemento-enamel junction" (AAP 2001). Mucogingival surgery was introduced by Friedman $^{1}$ in 1957 and described it as a surgical procedures performed to correct and eliminate anatomic, developmental, or traumatic deformities of the gingiva or alveolar mucosa. The prevalence of gingival recession is found in patients with both good and poor oral hygiene. Vigorous tooth brushing along with tooth malposition are the most frequent factors found to be associated with marginal tissue recession. Other local factors associated with root exposure are bony dehiscences, high frenal pull, poor oral hygiene and iatrogenic factors related to restorative and periodontal treatment procedures and some orthodontic movement of teeth. ${ }^{2}$

Gingival recession is common finding among population and many treatments have been implicated to cover the denuded root surfaces including laterally displaced flaps, coronally advanced flaps, free gingival grafts, connective-tissue grafts, acellular dermal matrix allografts and GTR may also be used. Selection of adequate surgical technique for root coverage depends on few local anatomic factors like size of the recession defect, width and height of keratinized tissue adjacent to the defect and vestibular depth or the presence of high frenum attachment while others are related to patients such as oral hygiene continuation, smoking. ${ }^{3}$

Grupe and warren ${ }^{4}$ introduced lateral pedicle graft (LPG) procedure in 1956 for coverage of denuded root surface as a shifting of full thickness donor flap laterally to cover an adjacent recession defect which provides sufficient esthetic results. Initially this procedure was known as the 'The lateral sliding flap' the procedure, which was later modified in incision design and renamed as 'laterally positioned flap. ${ }^{6}$

The present study was conducted to assess the clinical effectiveness of LPG procedure in the treatment of miller's class II gingival recession defects.

\section{Case Report}

A 35 years old female patient reported to the department of periodontology, FODS, King George's Medical University, Lucknow, UP,India, with the chief complaint of root hypersensitivity and receded gums from the past 1 year. On clinical assessment, Miller's class II recessions defect, with $5 \mathrm{~mm}$ vertical recession depth was found in relation to Mandibular right central incisor (Fig. 1). The patient was systemically sound without any oral abusive habits and had not undergone any periodontal handling in the last 6 months. A thorough explanation about surgical procedures was given to the patient and informed consent was obtained. Preparation of the patient included full mouth scaling and root planning and oral hygiene instructions.

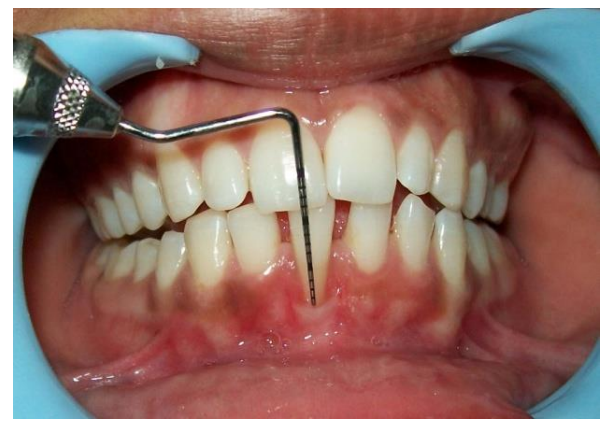

Fig. 1: Miller's class II recession irt 41 


\section{Surgical procedure}

Four week after phase-I therapy, the patient was subjected to surgical procedure. Prior to the surgery, routine laboratory investigations (hemoglobin, bleeding time, clotting time, total leukocyte count, differential leukocyte count, random blood sugar level, HIV and HbsAg) were done. The procedure was performed under local anesthetics of $2 \%$ lignocaine hydrochloride with $1: 80,000$ adrenaline.

Preparation of recipient site: After infiltration of local anaesthetics, incision were made with 15 no. blade on mesial and distal side of the recession defect removing only the margin and extending straight downward to a level slightly below the base of the defect. These incisions were connected by horizontal incision at the base of the defect. At the mesial side incision was externally beveled, whereas the distal margin was beveled internally. This gave a smoother gingival topography and the on lay effect in healing decreased the chances of development of a soft tissue slit at the site. The excised portion of the gingiva was then removed with curettes with no remnants of epithelial attachment remaining around the periphery of exposed root. At a distance approximately $3 \mathrm{~mm}$ from the wound edge, the attached gingiva was de-epithelised at the side opposite to the donor area and extending to a level approximately $3 \mathrm{~mm}$ apically and distally to create a recipient bed (Fig. 2)

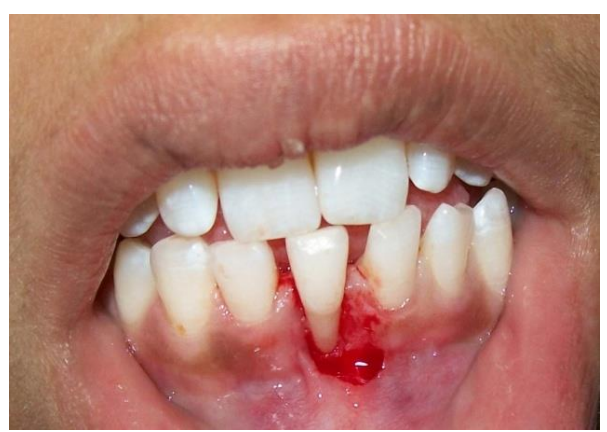

Fig. 2: Recipient site prepared

Preparation of donor site: Once the recipient site is prepared, width of denuded root surface was measured to facilitate correct surgical design of donor area. The pedicle graft size should be 1.5 times greater to the width of the defect to compensate for the shrinkage.

Incision was started at the distance of one papilla distal to the defect, in a direction straight downward beyond muco-gingival junction as far as necessary to provide adequate mobility to the flap. Now the full thickness flap was reflected, separating the attached gingiva from the underlying bone by blunt dissection down to its junction with alveolar mucosa. The flap was then positioned to cover the receptor site with no tension. (Fig. 3) Pressure was applied against the flap for 5 minutes to secure the good adaptation. It lied passively and remained pink prior to suturing. Cut back incision was placed at apical edge of the posterior vertical incision to further relieve the flap and to prevent button hole opening. The pedicle flap was sutured by using sling sutures and periodontal surgical dressing were placed to cover the surgical area (Fig. 4 and 5).

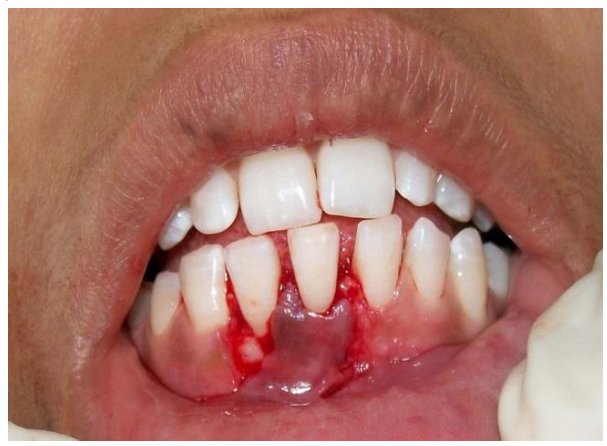

Fig. 3: Tension free lateral pedicle flap-repositioning

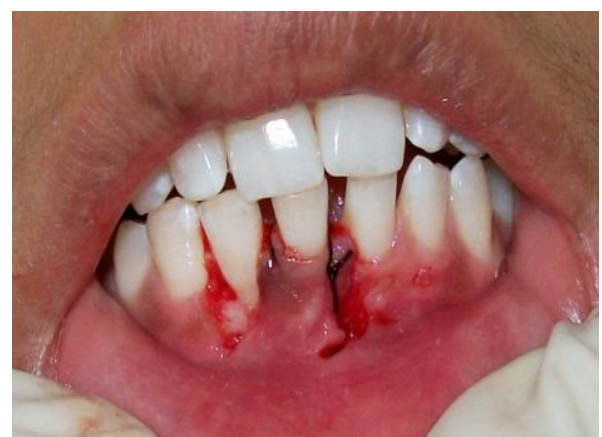

Fig. 4: Sling suture placed

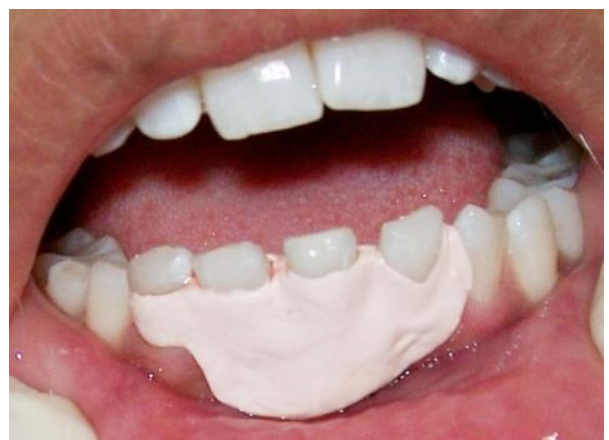

Fig. 5: Periodontal dressing placed

Antibiotics (Novamox LB $500 \mathrm{mg} 8$ hourly) and analgesic (ibuprofen $400 \mathrm{mg}$ trice daily) was prescribed for 5 days. The patient was asked to refrain from brushing in the operated area and to start $0.12 \%$ chlorhexidine gluconate mouth wash twice daily for two weeks. The patient was recalled 14 days postoperatively for suture removal. Then the patients were recall at interval of every one month to check for any plaque and or calculus deposition, which if present were removed by scaling. Then the final recordings were made at the end of 3 months (Fig. 6). 


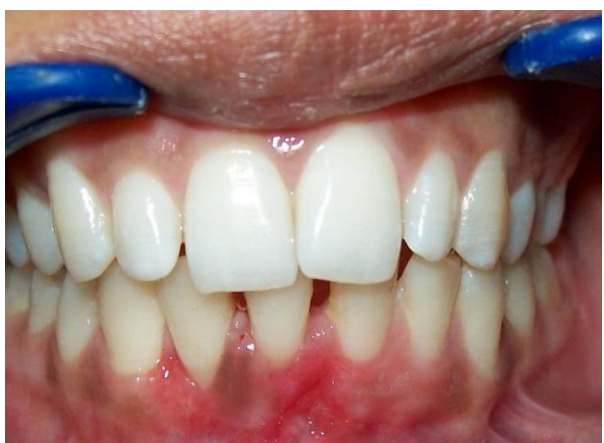

Fig. 6: Postoperative (At 3 months)

\section{Result}

At 3 months post-operatively, complete root coverage with tremendous tissue colour \& contour was obtained. Both donor and recipient site showed satisfactory healing with no signs of inflammation at final evaluation.

\section{Discussion}

The ultimate goal of any treatment aimed at root coverage is not only to restore the tissue margin at cementoenamel junction (CEJ) but to achieve new attachment of tissue to the root surface with regeneration of periodontium. The laterally displaced flap technique used to cover gingival recession is well documented and accepted. ${ }^{7}$ Introduced by Grupe and Warren 1956, it is a simple and convenient technique which offers many advantages over other procedures such as single surgical field, a good vascularized flap and a possibility of complete root surface coverage. ${ }^{8}$ Laterally positioned graft procedure involves shifting of donor tissue graft from an area adjacent to the recession defect to cover the denuded root surface.

In the present case, the patient with class II gingival recession was treated with LPG and the result showed significant root coverage with significant gain in attached gingiva. The LPG procedure performed in this case provide several advantages such as shield against root abrasion, reduction of dentin hypersensitivity and also absence of the second surgical site.

Moreover, a clinical study observed a statistically significant increase in attached gingiva with the LPF as compared to the CAF technique. ${ }^{9}$ Chambrone $^{10}$ et al.., (2009) and Santana ${ }^{11}$ et al., (2010) achieved 93.8\% and $95.5 \%$ of the root coverage respectively by laterally displaced flap. The most important factor determining the success of laterally displaced flap is the proper case selection, with sufficient donor site in terms of thickness and height.

\section{Conclusion}

In present case report complete coverage of root surface was obtained by LPG technique, so it can be concluded that LPG procedure can be a promising technique for treatment of isolated gingival defect.

\section{References}

1. Friedman N. Mucogingival surgery. Tex Dent $J$ 1957;75:358-62.

2. Caudill RF, Oringer RJ, Langer L et al. Esthetic Periodontics (periodontal plastic surgery), In: Wilson TG, Kornman KS eds. Fundamentals of Periodontics, Quintessence Publishing Co. Inc 1996:497-518.

3. Zucchelli G, Testori T, De Sanctis M. Clinical and anatomical factors limiting treatment outcomes of gingival recession: A new method to predetermine the line of root coverage. J Periodontol 2006;77:714-21.

4. Grupe HE, Warren RF. Repair of gingival defects by sliding flap operation. J Periodontol 1956; 27:92-5.

5. Roccuzzo M, Bunino M, Needleman I, Sanz M. Periodontal plastic surgery for treatment of localized gingival recessions: A systematic review. J Clin Periodontol 2002;29:178-94.

6. Grupe H. Modified technique for the sliding flap operation. Periodontol 1966;37:491-95.

7. Pfeifer JS, Heller R. Histologic evaluation of full and partial thickness laterally reposiotioned flap: A pilot study. J Periodontol 1971;42:331-3.

8. Georgiev T. Combining a laterally moved flap in closing local gingival recession with the use of straumann emdogain gel. Journal of IMAB- Annual Proceeding (scientific papers) 2010;16:41-2.

9. De waal H, Kon S, Ruben MP. The laterally positioned flap. Dent Clin North Am 1988;32(2):267-85.

10. Chambrone L, Chambrone D, Pustiglioni FE, Chambrone LA. The Influence of Tobacco Smoking on the Outcomes Achieved by Root-Coverage Procedures. J Am Dent Assoc 2009; 140(3):294-306.

11. Santana RB, Furtado MB, Mattos CML and Fonseca E. Clinical Evaluation of Single-Stage Coronally Advanced Versus Rotated Flaps in the Treatment of Gingival Recessions. J Periodontol 2010;81(4): 485-92. 